\title{
Caution with Hair Dye: Foreign Body Skull Erosive Granuloma from Exogenous Hair Pigmentation
}

\section{Dear Editor,}

Modification of hair colour with the use of hair dye is common. Allergic reactions and contact dermatitis and are common side effects of hair dye on the scalp that have been described in literature. ${ }^{1,2,4}$ Scalp nodules related to pigments of hair dye is a rare condition, with only a few isolated case reports from overseas. ${ }^{3}$

In this letter, we will present a rare case of a 40 -yearold gentleman who presented to us with a scalp lump, with associated skull bone erosion seen on the radiological imaging. Final histology from surgical excision concluded a foreign body granulomatous reaction to hair dye substances.

\section{Case Presentation}

The patient who had dyed his hair a few months earlier, presented to our Department of Neurosurgery with a 2-month history of a tender left parietaloccipital scalp lump. He had no history of scalp trauma. There were no previous infections or tattoos at the area, and the patient did not have a family history for dermatological malignancies. Physical examination revealed a $2 \mathrm{~cm}$ tender and hard lump at the left parietal-occipital scalp area.

A computed tomography (CT) scan of the head confirmed an enhancing lesion at the index location with erosion of the outer cortex of the underlying skull (Figures 1 and 2). The initial differential diagnosis for the condition included that of a foreign body granuloma, sebaceous cyst, nodular melanoma, dermatofibroma and histiocytosis.

The patient underwent an excision of the left parietal-occipital scalp lesion. Intraoperatively, we noted a hard, rubbery, grey-coloured flat-based lesion beneath the galea at the index location, measuring approximately $1.5 \mathrm{~cm} \times 1.5 \mathrm{~cm}$. The epicentre of this lesion was attached to and eroding the outer cortex of the underlying bone, leaving an indentation of the outer cortex. The lesion was separated from the surrounding bone and excised completely using sharp dissection. It was sent for both frozen section and formal histology.

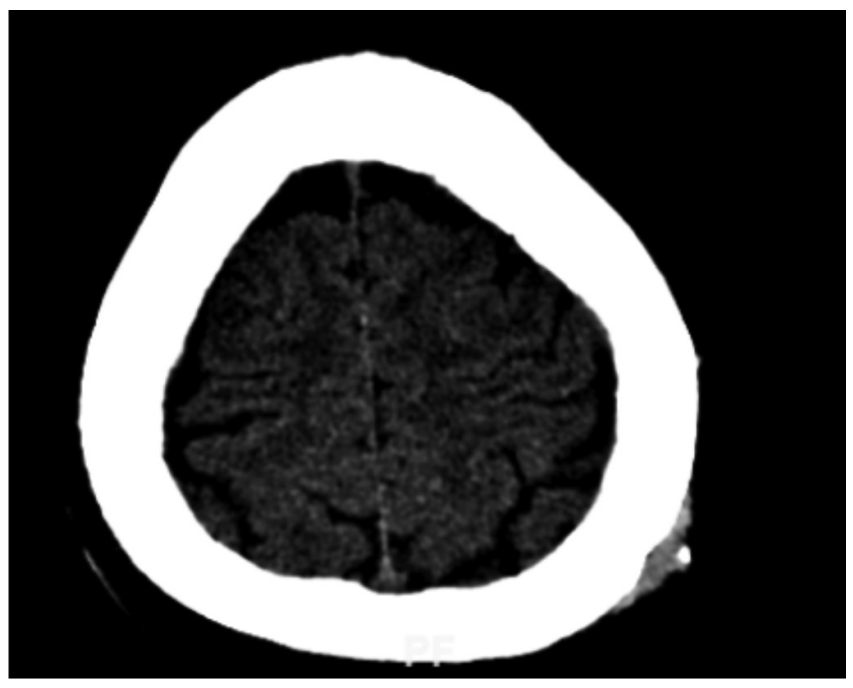

Fig. 1. Contrasted CT scan of the head showing the contrast enhancing left parietal scalp lesion with outer cortex skull erosion.

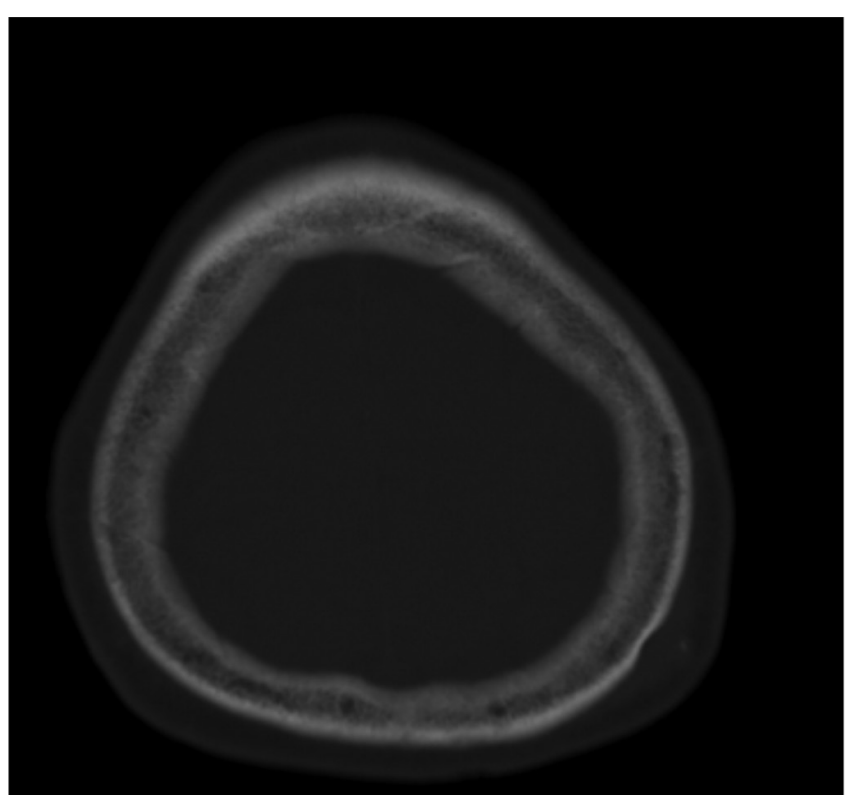

Fig. 2. Bone window of the same CT scan showing the outer cortex of skull erosion at the left parietal area. 
The intraoperative frozen section revealed aggregates of atypical oval to spindle cells. In view of the frozen section findings, which suggested a possibility of malignancy, a small craniotomy was fashioned around the margins of the lesion where it had eroded the skull. The inner cortex of the bone flap was noted to be normal. The underlying dura was normal with no breach.

The craniotomy defect was repaired with a titanium mesh secured with micro-screws and this mesh was overlaid with a thin layer of tobramycin antibiotic cement.

The patient had an uneventful recovery and the final histology confirmed features indicative of a foreign body granulomatous reaction to exogenous blackish material, which may represent exogenous pigment or dye. A diagnosis was made by the histopathologist of foreign body granulomatous reaction in the presence of known hair dye substances.

The patient remained well on follow-up with no recurrence of the scalp lump. The surgical wound healed well and he was discharged from regular follow-up.

\section{Discussion}

Histologically, granulomas are aggregates of mononuclear inflammatory cells or modified macrophages, which are usually surrounded by a rim of lymphocytes and often contain a few giant cells. Granulomas typically form to protect the host from persistent inflammatory stimuli which, if ongoing, may produce locally inflammatory and destructive effects. ${ }^{5}$

Primary granuloma formation can be classified into autoimmune, infectious, idiopathic and hereditary causes. Secondary granuloma formation in the scalp can be a result of foreign body implantation and chemical exposure, such as that of the hair dye in this case.

Hair dyes are classified as either "semi-permanent" or "permanent" dyes according to their ability to permeate the hair shaft. The main difference between the two types dyes lie in their capacity to either penetrate the hair follicle cortex permanently, or to stay shallow on the cuticle surface and be washed after a few rounds of shampooing. To overcome the cuticle and reach the cortex layer, the product must have an alkaline $\mathrm{pH}$ able to open the scales of the hair follicle.
Most permanent dyes use ammonia to increase the $\mathrm{pH}$. The permanent dye works by an oxidation reaction that allows the pigments to get inside the cortex of the hair. These pigments are: paraphenylenediamine, paratoluenediamine, and paraaminophenol, with hydrogen peroxide to liberate oxygen. Once inside the cortex, they combine with aniline dyes to produce the required colour molecules. ${ }^{2}$

Semi-permanent dyes, on the other hand, do not contain ammonia or ethanolamine. They do however also contain hydrogen peroxide, resorcinol and paradyes. The concentration of hydrogen peroxide in semi-permanent dyes is lower $(2 \%)$ as compared to that of permanent hair dyes $(6 \%){ }^{2}$

While foreign body type secondary scalp granuloma, in association with lipid material (mineral oil) deposited in tissues used for cosmetic body contour augmentation was reported previously, ${ }^{6}$ modification of hair colour with the use of hair dye is now the more common trend. In this case, the patient had a short history of hair dye exposure, coupled with rapid growth and partial involvement of the outer cortex of the skull. In the presence of the frozen section report of atypia, this does raise the concern of malignancy with skull bone involvement. Such concern would augment the clinical context from a simple cosmetic scalp lump open resection surgery, to a more extensive surgery requiring part of cranial bone removal (craniectomy).

\section{Conclusion}

This is, to our knowledge, the first report of granulomatous inflammation of the scalp, causing erosion of the skull bone after the use of colouring hair dye. Hair dye granulomas may mimic malignant scalp lesion, as demonstrated in this case. Anamnesis is the physician's most important basis of diagnosis, with skin biopsy being performed in suspicious lesions. Our case is unusual due to the uncommon aetiology and the atypical location. The histological findings were decisive to making the correct diagnosis and treatment.

Following this case report, we would like to sound a note of caution for those who consider using such hair dyes, and alert our medical colleagues to seek a history of hair dye usage when patients present to them with suspicious scalp lumps or pigmented scalp lesions. 


\section{REFERENCES}

1. Søsted H, Rustmeyer T, Gonçalo M, Bruze M, Goossens A, Giménez-Arnau AM, et al. Contact allergy to common ingredients in hair dyes. Contact Dermatitis. 2013;69(1):32-9

2. Kim KH, Kabir E, Jahan SA. The use of personal hair dye and its implications for human health. Environ Int. 2016;89-90:222-7.

3. Nallayici EG, Kroft, Hogenes, Schreurs. Case Report: A Nodule on the Scalp with Exogenous Pigment. Clin Surg. 2017; 2: 1423.

4. Patra AP, Shaha KK, Rayamane AP, Dash SK, Mohanty MK, Mohanty S. Paraphenylenediamine containing hair dye: an emerging household poisoning. Am j Forensic Med Pathol. 2015;36(3):167-71.

5. Kaye P. Granulomatous diseases. Int J Exp Pathol 2000; 81(5):289-290.
6. Husein-Elahmed H, Soriano-Hernandez MI, Aneiros-Cachaza J, Ruiz-Carrascosa JC, Naranjo-Sintes R. Ulceration of the scalp: lipogranuloma induced by industrial oils in a decorator woman. Ann Acad Med Singapore. 2012 Mar;41(3):132-3.

David SK $\underline{\text { Mak }},{ }^{1}$ MBBS (Ireland), MRCS (Edinburgh),

Ying Khai Peter Hwang, ${ }^{1}$ MBBS, FRACS (Neurosurgery), FRCS (Edinburghand Glasgow)

${ }^{1}$ Department of Neurosurgery, National Neuroscience Institute, Tan Tock Seng Hospital, Singapore

Address for Correspondence: Dr David SK Mak and Dr Hwang Ying Khai, Department of Neurosurgery, National Neuroscience Institute, Tan Tock Seng Hospital, 11 Jalan Tan Tock Seng, Singapore 308433 